\title{
A Complex Suicide by Ligature Strangulation and Hanging Using Two Different Ligatures
}

Kunasilan Subramaniam ${ }^{1}$, Chandroth Navin Pankajakshan ${ }^{2}$

${ }^{1}$ National Institute of Forensic Medicine, Hospital Kuala Lumpur, Kuala Lumpur, Malaysia, ${ }^{2}$ Department of Forensic Medicine, Hospital Pulau Pinang, George Town, Malaysia

Received: July 12, 2019

Revised: August 23, 2019

Accepted: August 29, 2019

\section{Correspondence to}

Kunasilan Subramaniam

National Institute of Forensic

Medicine, Hospital Kuala Lumpur,

Jalan Pahang, 50586 Kuala Lumpur,

Malaysia

Tel: +60-326155299

Fax: $+60-326941422$

E-mail:kuna0921@yahoo.com
Hanging is the most common method of suicide in Malaysia. However, hanging in combination with suicidal ligature strangulation is uncommon. The victim is a 31-year-old man, with no previous medical or psychiatric disorders. He accomplished self-strangulation using a shoelace and hanging himself with a High-Definition Multimedia Interface cable. Three loops of a shoelace ligature were present around the neck. A single knot was present on the front, and a double knot at the back of the neck. Internal examination of the neck revealed small bilateral hemorrhages of the sternocleidomastoid muscles. No laryngeal cartilage or hyoid bone fractures were observed. No other evidence of injury was noted other than moderate pulmonary edema. The post-mortem toxicology results were negative. The cause of death was ascertained as neck compression due to ligatures. Scene assessment and post-mortem findings concur with suicide. This report describes an unusual case of suicidal ligature strangulation, in combination with hanging using two different ligatures.

Key Words: Hanging; Ligature strangulation; Post-mortem; Suicide

\section{Introduction}

According to the statistics of the Malaysian National Suicide Registry, hanging is the most common method of suicide in Malaysia [1]. However, hanging in combination with suicidal ligature strangulation is rather uncommon. A ligature strangulation case should be considered as a homicide unless proven otherwise. This article reports a case of suicidal strangulation by ligature, coupled with hanging, of an individual who had no previous medical or psychiatric disorder. He initiated self-strangulation using a shoelace, followed by hanging using a High-Definition Multimedia Interface (HDMI) cable. Scene assessment and post-mortem findings concurred with suicide. The unusual case of suicidal ligature strangulation in combination with hanging using two different ligatures is reported in this case.

\section{Case Report}

The deceased is a 31-year-old man of Chinese ethnicity, who was single and lived with his parents in a twostory house. He was a diploma graduate and worked for a metal company. He was last seen alive at 8:00 pm after returning home from dinner with his parents. The next day, at about 3:00 pm, his father noticed that his son was not yet awake and discovered that his room door was locked from the inside. Having received no 
response after several knocks, the parents decided to break into the room. They found their son suspended from the ceiling with an HDMI cable (Fig. 1). The police later arrived at the scene and brought the body down by cutting the HDMI cable. Further examination of the immediate surroundings revealed that there was a ladder next to the deceased which is believed to have been used to tie the cable to the ceiling. No pornographic material or other sexual paraphernalia was found in the room. Although the room appeared to be unkempt, it did not present any evidence of struggle. Death was confirmed by the attending paramedics before the body was transported to the mortuary by the police for a post-mortem examination.

A post-mortem examination was carried out the next day, with an estimated post-mortem interval of 38 hours from the time he was last seen alive. An external examination revealed fixed hypostasis at the back and lower limbs. There was marked facial congestion and moderate petechial bleeding on the conjunctivae and skin of the face which was sharply demarcated by the strangulation region. The hanging ligature mark at the upper part of the neck, which was consistent with the HDMI cable, was present as a linear furrow measuring $1 \mathrm{~cm}$ wide. This parchmented abrasion was found to be directed towards the back of the ears before waning off at the level of the temple on both sides. Just below the cable ligature mark, three loops of a shoelace ligature were present on the neck. A single knot was present on the front, and a double knot at the back, of the neck (Fig. 2). The ligature marks were present in the form of a continuous patterned horizontal groove which completely encircled the neck: the mean width was 0.5 $\mathrm{cm}$ but it measured $1.5 \mathrm{~cm}$ at the anterior aspect of the neck (Fig. 3). No other external injuries were identified on his body. Internal examination of the neck revealed small hemorrhages on the bilateral sternocleidomastoid muscles beneath the external ligature mark. Apart from that, there were no hemorrhages seen on the strap muscles of the neck. There were no laryngeal cartilage or hyoid bone fractures. No other evidence of injury was noted other than moderate pulmonary edema. Post-mortem toxicology reports showed the presence of $38 \mathrm{mg} / \mathrm{dL}$ of ethyl alcohol in the blood. However, no drugs were detected. The cause of death was therefore ascertained as neck compression due to ligatures.

\section{Discussion}

Suicide is increasing in numbers globally, with hanging being one of the most common methods encountered [2]. The constricting force around the neck which is derived from the gravitational force of the body weight causes death while hanging. This is in contrast with deaths due to suicidal ligature strangulations, which occur when a constant force is maintained around the neck to obstruct the venous blood flow [3]. Polson demonstrated that a force of only $3.2 \mathrm{~kg}$ is necessary to occlude arterial vessels, whereas a force of $2 \mathrm{~kg}$ is enough to occlude the venous system [4]. Thus, a simple ligature, like an elastic band, can cause death by strangulation, which has in fact been reported in the literature [5]. Determination of the cause of death in cases of ligature strangulation is a challenge for crime

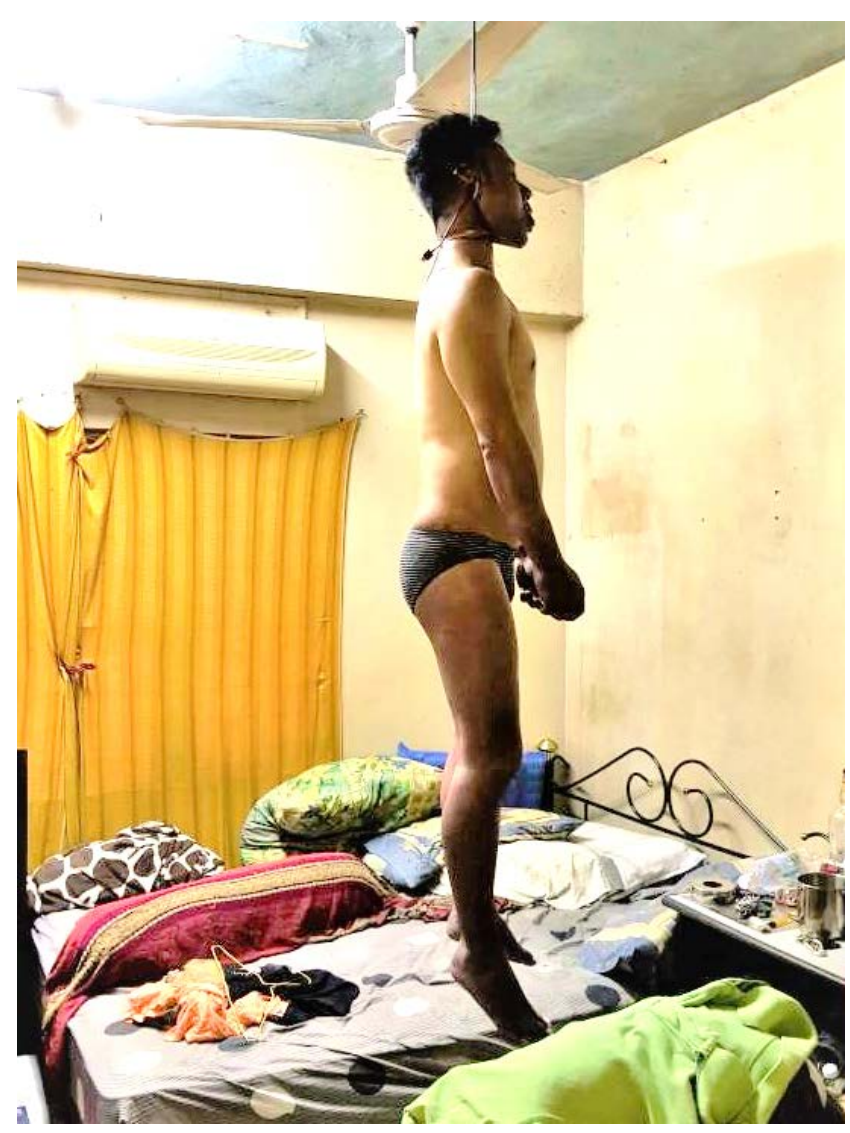

Fig. 1. Deceased is shown suspended from the ceiling with a HighDefinition Multimedia Interface cable. Note the attached shoelace used as a ligature for strangulation. 
investigators and forensic pathologists alike. Ligature strangulation is often encountered in homicides, with suicidal ligature strangulation being relatively rare. The task of differentiating self-strangulation from homicidal strangulation is onerous, given the fact that there are no pathognomonic features to distinguish one from the other [6].

As rare as it is, there have been cases of suicidal ligature strangulations reported previously. Three such papers have described the absence of hyoid bone or thyroid cartilage fractures, but minimal hemorrhage of the strap muscles [7-9]. This was in contrast with one case described by Demirci et al. [10], in which there was a fracture and ecchymosis of the left upper horn of the thyroid cartilage. Given the findings of the case at hand, the shoelace had left a more prominent mark than the HDMI cable, which together with the facial congestion and conjunctival petechiae may indicate a homicide. However, the internal findings revealed that, other than the trivial hemorrhages of the sternocleidomastoid

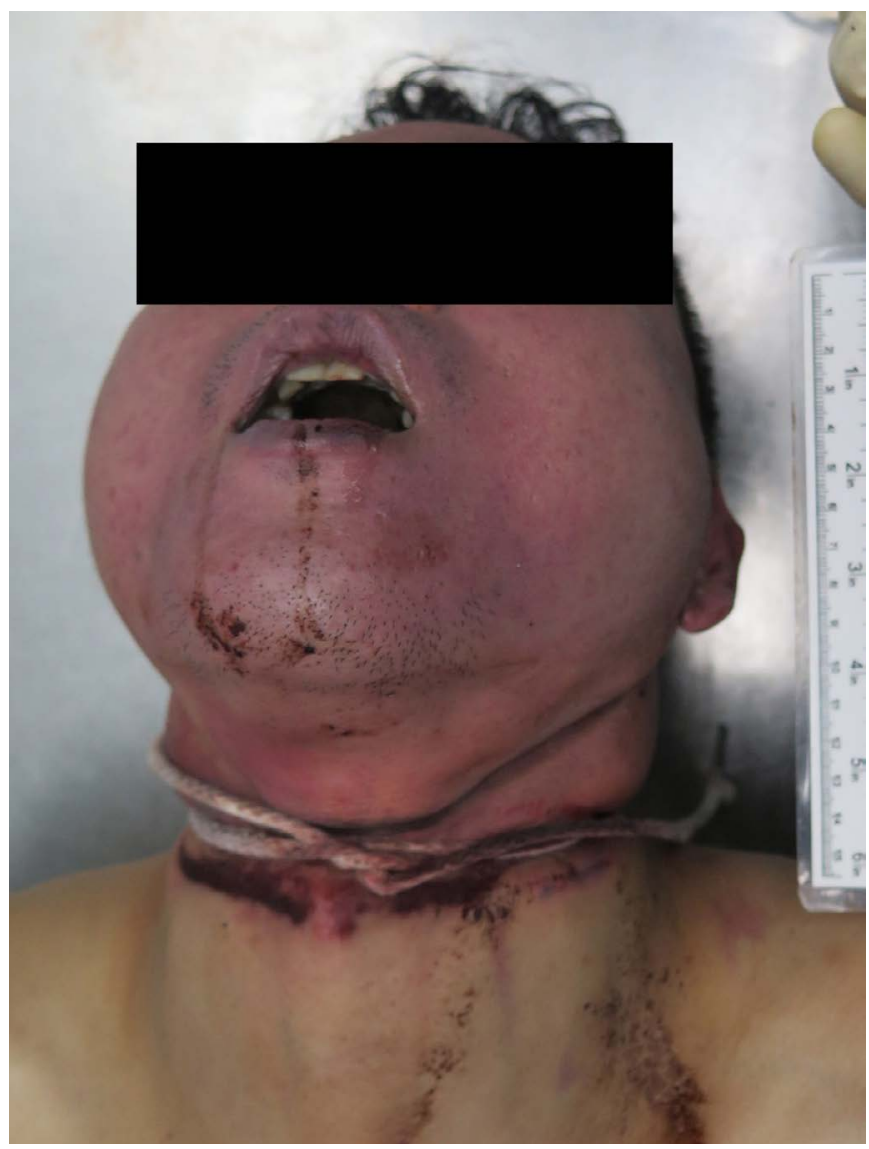

muscles, the laryngeal cartilage and hyoid bone were left uninjured, which is consistent with suicide. Fixed post-mortem lividity mainly in the lower limbs and forearm is suggestive that the body was suspended in a prolonged vertical position after death [3]. This is also consistent with the timeline derived from the investigation report.

In 1961, Polson [4] identified several presumptive techniques for suicidal strangulation. Later, various authors presented suicidal ligature strangulation cases describing similar methods with some modifications $[8,11,12]$. Neither multiple ligatures nor repeated knotting is strongly indicative of homicidal action [6] The use of more than one ligature as well as multiple turns, up to 20 , has been repeatedly reported in suicides. This case presents with similar findings, whereby there was a shoelace which had two turns around the neck, coupled with an HDMI cable used for the act of suspension.

The location of the knot is crucial in determining

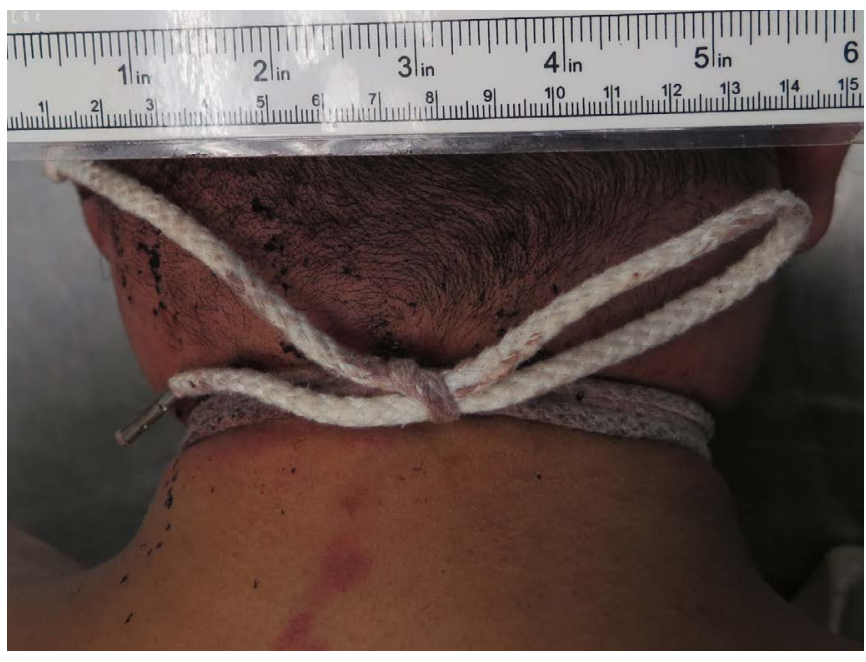

B

Fig. 2. Three loops of a shoelace ligature were present on the neck. $A$ single knot was present on the front $(\mathbf{A})$, and double knot at the back, of the neck (B). 
whether the deceased was able to reach the knot. A literature review showed that the posterior position of the knot is rare in suicidal strangulation [6]. Therefore, the self-strangulation technique in the case at hand is unique compared to previously reported cases. The anterior knot is an overhand knot produced by crossing two ends of a ligature over each other in the front [13]. It is unstable in nature unless the ligature has a rough texture. The posterior knot was a figure eight on top of the overhand knot. This knot stabilized the ligature. Both knots could have been made by the deceased himself. Therefore, in this case, the ligature strangulation could be suicidal.

On the other hand, the application of a tight ligature around the neck is a general finding in homicidal strangulation which warrants a thorough examination to confirm or refute it as the manner of death [4]. In this case, suicide was considered as the manner of death, taking into account his father's witness statement and the findings at the scene, which showed that the door was locked from the inside and there were no signs of violence. The postulated sequence of events is that the deceased had first tied the shoelace tightly at the back of his neck before he hung himself with the HDMI cable, which was tied to a hook attached to the ceiling. The underlying shoelace ligature, fixed by a figure-eight knot, exerted constant pressure around the neck of the deceased, even after unconsciousness, and obstructed venous flow long enough to cause cerebral hypoxia and ultimately death. Moreover, the resulting

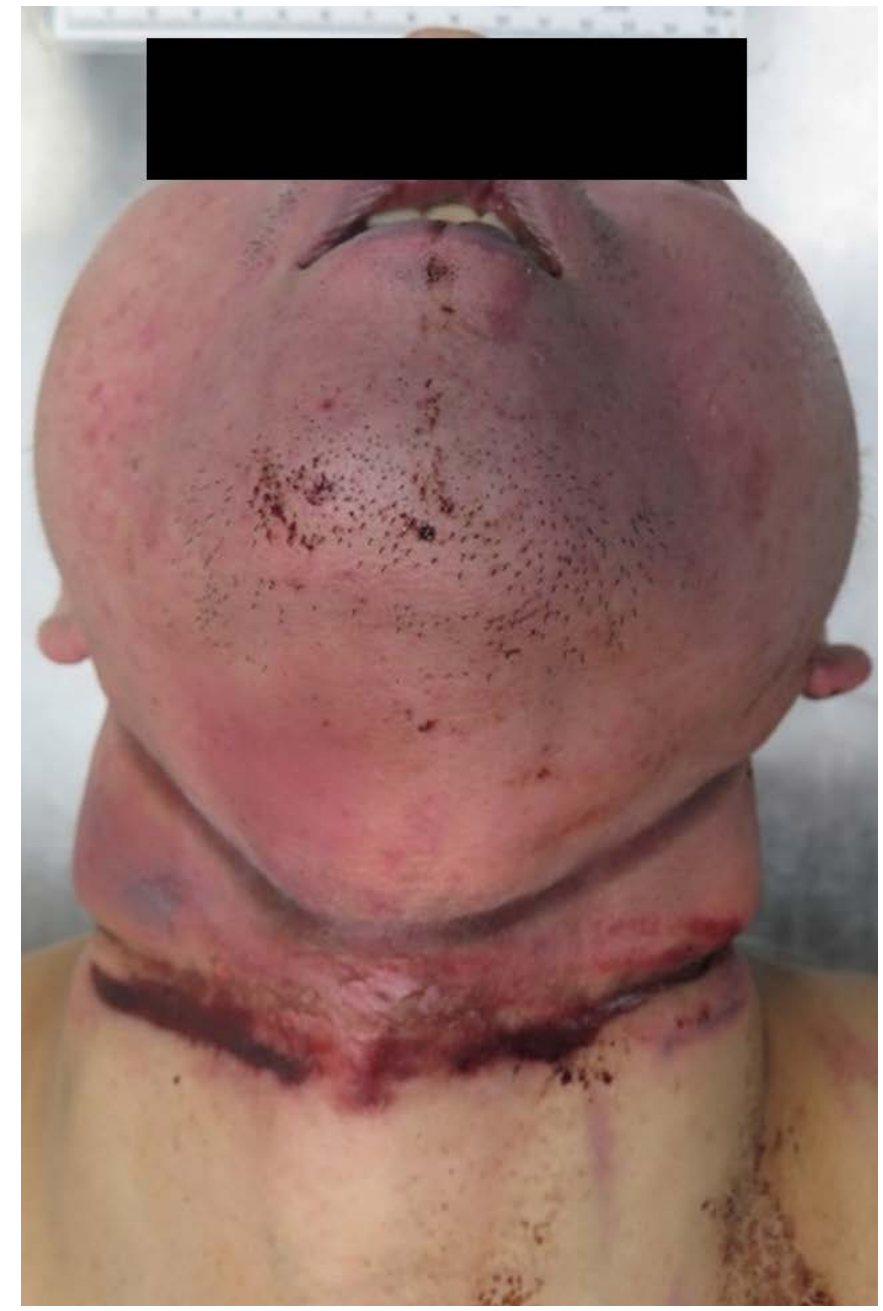

A

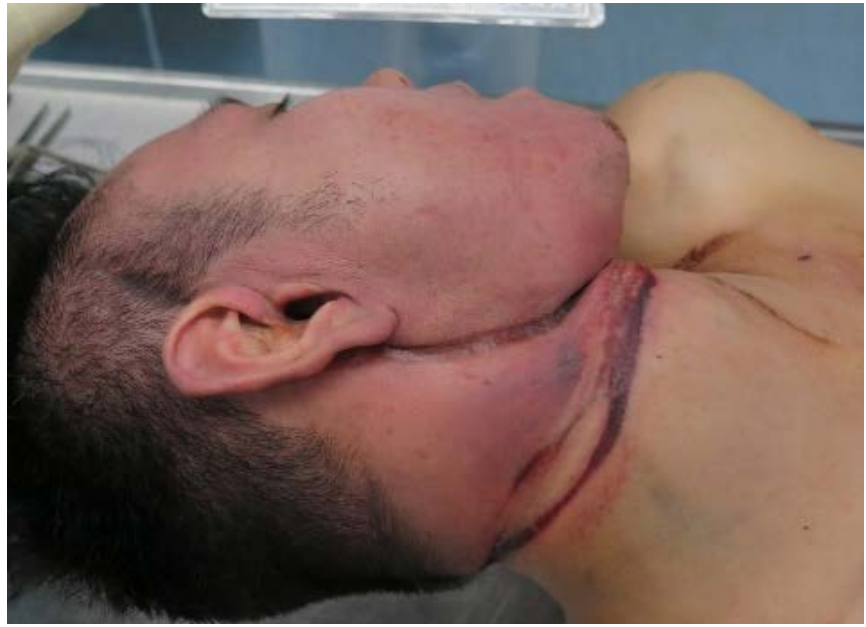

B

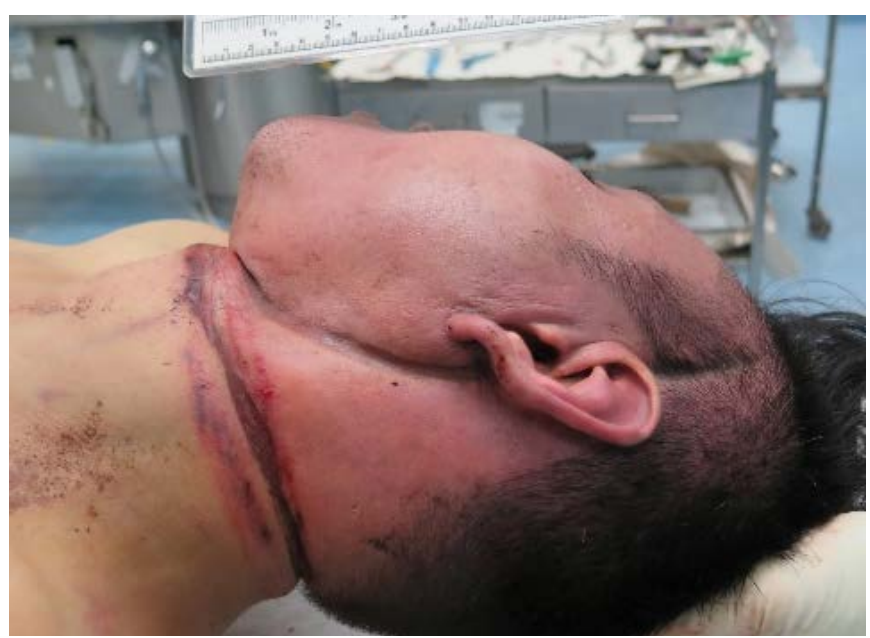

C

Fig. 3. Two well-demarcated ligature marks can be seen on the neck. The view from the front $(\mathbf{A})$, from the right (B), and from the left $(\mathbf{C})$ shows the shoelace ligature marks were present in the form of a continuous patterned horizontal groove, which completely encircled the neck. 
$38 \mathrm{mg} / \mathrm{dL}$ blood ethyl alcohol level was not enough to cause incapacitation and suggests full capacity of the deceased to accomplish the act of strangulation.

The present case emphasizes the importance and needs to consider multiple available evidentiary issues to reach conclusions about the cause of death. One single finding may not always definitively determine homicide or suicide in suspicious deaths. Analysis of the number of ligatures, position of the knots, number of knots and turns, characteristics of ligature marks, and the absence of defense injuries in ligature strangulations cases is crucial. Detailed documentation of these features at the scene or in the autopsy room is vital to differentiate between suicide and homicide. Questions as to whether the subject could reach the knot alone and the possibility of self-inflicted injuries must also be addressed. In cases of ligature-related asphyxia, documentation of the characteristics of the ligature and the knot tied is essential. Therefore, the noose should not be removed, and the knots should be left intact. Proper coordination between the crime investigators and the forensic pathologist can provide a correct interpretation of the findings and quickly clarify the manner of death in these particularly complex cases.

ORCID: Kunasilan Subramaniam: https://orcid.org/00000003-3011-6576; Chandroth Navin Pankajakshan: https://orcid.org/0000-0002-9727-845X

\section{Conflicts of Interest}

No potential conflict of interest relevant to this article was reported.

\section{Acknowledgments}

The authors thank the Director General of Health Malaysia for his permission to publish this article. Also, we would like to express our appreciation to the Director of Hospital Kuala Lumpur and Director of National Institute of Forensic Medicine, Malaysia for allowing the use of resources throughout the study.

\section{References}

1. Hayati AN, Kamarul AK. National Suicide Registry Malaysia (NSRM). Med J Malaysia 2008;63 Suppl C:50-4.

2. Varnik P. Suicide in the world. Int J Environ Res Public Health 2012;9:760-71.

3. Di Maio VJ, Di Maio D. Forensic pathology. 2nd ed. Boca Raton: CRC Press; 2001. p. 23, 245-75.

4. Polson CJ. Strangulation: accident, suicide or homicide.J Forensic Sci Soc 1961;1:79-8.

5. Tzimas I, Bajanowski T, Pollak S, et al. Suicidal ligature strangulation using gymnastics bands. Int J Legal Med 2014;128:313-6.

6. Maxeiner H, Blockholdt B. Homicidal and suicidal ligature strangulation: a comparison of the post-mortem findings. Forensic Sci Int 2003;137:60-6.

7. Badiadka KK, Kanchan T, D'Souza DH, et al. An unusual case of self-strangulation by ligature.J Forensic Leg Med 2012;19:434-6.

8. Atilgan M. A case of suicidal ligature strangulation by using a tourniquet method. Am J Forensic Med Pathol 2010;31:85-6.

9. Pramod Kumar GN, Arun M, Manjunatha B, et al. Suicidal strangulation by plastic lock tie.J Forensic Leg Med 2013;20:602.

10. Demirci S, Dogan KH, Erkol Z, et al. Suicide by ligature strangulation: three case reports. Am J Forensic Med Pathol 2009;30:369-72.

11. Gaur JR, Verma RK, Thakur GC. Suicidal strangulation. Med Sci Law 1992;32:55-6.

12. Palmiere C, Risso E, van Hecke 0 , et al. Unplanned complex suicide by self-strangulation associated with multiple sharp force injuries: a case report. Med Sci Law 2007;47:269-73.

13. Ashley CW. The Ashley book of knots. New York: Doubleday, Doran \& Co.; 1993.p. 220. 